Commun. Korean Math. Soc. 21 (2006), No. 1, pp. 101-115

\title{
LOCALIZATION PROPERTY AND FRAMES II
}

\author{
Young-Hwa Ha AND JU-YeON RYu
}

\begin{abstract}
Localization of sequences with respect to Riesz bases for Hilbert spaces are comparable with perturbation of Riesz bases or frames. Gröchenig first introduced the notion of localization. We introduce more general definition of localization and show that exponentially localized sequences and polynomially localized sequences with respect to Riesz bases are Bessel sequences. Furthermore, they are frames provided some additional conditions are satisfied.
\end{abstract}

\section{Introduction}

The main feature of a basis $\left\{f_{i}\right\}_{i=1}^{\infty}$ for a Hilbert space $H$ is that every $f \in H$ can be represented as a convergent series in terms of the elements $f_{i}$, that is, $f=\sum_{i=1}^{\infty} c_{i} f_{i}$ with unique coefficients $c_{i}$. A frame is also a sequence $\left\{f_{i}\right\}_{i=1}^{\infty}$ in $H$ which allows every $f \in H$ to be written as a series. But, it is overcomplete and so the series representations need not be unique. This redundancy may be useful, for example, in applications to noise reduction or for reconstruction from lossy data. In addition, construction of a frame is easier and more flexible than construction of a basis.

A sequence $\left\{f_{i}\right\}_{i=1}^{\infty}$ of elements in a Hilbert space $H$ is called a Bessel sequence if there exists a constant $B<\infty$ such that for every $f \in H$,

$$
\sum_{i=1}^{\infty}\left|\left\langle f, f_{i}\right\rangle\right|^{2} \leq B\|f\|^{2}
$$

Received August 1, 2005. Revised October 4, 2005.

2000 Mathematics Subject Classification: 42C15, 46E99, 46B15.

Key words and phrases: frame, Bessel sequence, Riesz basis, exponentially localized, polynomially localized, localization of frames. 
A Bessel sequence $\left\{f_{i}\right\}_{i=1}^{\infty}$ is called a frame if it satisfies an additional condition : there exists a constant $A>0$ such that for every $f \in H$,

$$
A\|f\|^{2} \leq \sum_{i=1}^{\infty}\left|\left\langle f, f_{i}\right\rangle\right|^{2} .
$$

The numbers $A$ and $B$ are called a lower and an upper frame bound of the frame, respectively. If $\left\{f_{i}\right\}_{i=1}^{\infty}$ is a frame, then it is well-known that for every $f \in H, f=\sum_{i=1}^{\infty} c_{i} f_{i}$ for some coefficients $\left\{c_{i}\right\}_{i=1}^{\infty} \in l^{2}(\mathbb{N})$.

A sequence $\left\{f_{i}\right\}_{i=1}^{\infty}$ of elements in a Hilbert space $H$ is called a Riesz basis if it is complete and there exist positive constants $A$ and $B$ such that for every $\left\{c_{i}\right\}_{i=1}^{\infty} \in l^{2}(\mathbb{N})$,

$$
A \sum_{i=1}^{\infty}\left|c_{i}\right|^{2} \leq\left\|\sum_{i=1}^{\infty} c_{i} f_{i}\right\|^{2} \leq B \sum_{i=1}^{\infty}\left|c_{i}\right|^{2} .
$$

The numbers $A$ and $B$ are called a lower and an upper Riesz bound of the Riesz basis, respectively. If $\left\{f_{i}\right\}_{i=1}^{\infty}$ is a Riesz basis for a Hilbert space $H$, then there exists a unique sequence $\left\{g_{i}\right\}_{i=1}^{\infty}$ in $H$ such that for every $f \in H, f=\sum_{i=1}^{\infty}\left\langle f, g_{i}\right\rangle f_{i}$. Such a sequence $\left\{g_{i}\right\}_{i=1}^{\infty}$ is called the dual Riesz basis of $\left\{f_{i}\right\}_{i=1}^{\infty}$. There are well-known characterizations for Riesz bases (see [11]). For instance, a Riesz basis is an $\omega$-independent frame.

Gröchenig introduced a concept of localization of sequences in [5] and [7]. In the next section, we give more general definition of localization. To compare with our definition, let us give Gröchenig's definition of localization in [7]. A sequence $\left\{f_{i}\right\}_{i=1}^{\infty}$ in a Hilbert space $H$ is called exponentially localized with respect to a Riesz basis $\left\{g_{i}\right\}_{i=1}^{\infty}$ if there exist positive constants $\alpha$ and $C$ such that $\left|\left\langle f_{i}, g_{j}\right\rangle\right| \leq C e^{-\alpha|i-j|}$ and $\left|\left\langle f_{i}, \tilde{g}_{j}\right\rangle\right| \leq C e^{-\alpha|i-j|}$ for all $i, j \in \mathbb{N}$, where $\left\{\tilde{g}_{i}\right\}_{i=1}^{\infty}$ is the dual Riesz basis of $\left\{g_{i}\right\}_{i=1}^{\infty}$. Similarly, a sequence $\left\{f_{i}\right\}_{i=1}^{\infty}$ in a Hilbert space $H$ is called polynomially localized with respect to a Riesz basis $\left\{g_{i}\right\}_{i=1}^{\infty}$ if there exist positive constants $s$ and $C$ such that $\left|\left\langle f_{i}, g_{j}\right\rangle\right| \leq C(1+|i-j|)^{-s}$ and $\left|\left\langle f_{i}, \tilde{g_{j}}\right\rangle\right| \leq C(1+|i-j|)^{-s}$ for all $i, j \in \mathbb{N}$, where $\left\{\tilde{g}_{i}\right\}_{i=1}^{\infty}$ is the dual Riesz basis of $\left\{g_{i}\right\}_{i=1}^{\infty}$. It can be shown that if a sequence $\left\{f_{i}\right\}_{i=1}^{\infty}$ is exponentially or polynomially localized with respect to a Riesz basis then it is a Bessel sequence (see [8]).

The results in this paper are extensions of the results in our previous paper [10]. In [10], we found some sufficient conditions which guarantee that localized sequences are frames. In this paper, we consider the same problem with generalized definitions of localization. 


\section{Definitions}

DEFINITION 2.1. A sequence $\left\{I_{i}\right\}_{i=1}^{\infty}$ is called a sequence of indexing sets if $I_{i}$ are finite and disjoint and $\mathbb{N}=\bigcup_{i=1}^{\infty} I_{i}$.

Definition 2.2. A sequence $\left\{f_{i}\right\}_{i=1}^{\infty}$ in a Hilbert space $H$ is called exponentially localized with respect to a Riesz basis $\left\{g_{i}\right\}_{i=1}^{\infty}$ if there exist a sequence of indexing sets $\left\{I_{i}\right\}_{i=1}^{\infty}$, positive constants $r<1$, and $C$ such that

$$
\left|\left\langle f_{k}, g_{j}\right\rangle\right| \leq \frac{C}{\sqrt{n}_{i}} r^{|i-j|}
$$

and

$$
\left|\left\langle f_{k}, \tilde{g}_{j}\right\rangle\right| \leq \frac{C}{\sqrt{n}_{i}} r^{|i-j|}
$$

for all $i, j \in \mathbb{N}$ and $k \in I_{i}$, where $\left\{\tilde{g}_{i}\right\}_{i=1}^{\infty}$ is the dual Riesz basis of $\left\{g_{i}\right\}_{i=1}^{\infty}$ and $n_{i}$ is the cardinality of $I_{i}$.

Definition 2.3. A sequence $\left\{f_{i}\right\}_{i=1}^{\infty}$ in a Hilbert space $H$ is called polynomially localized with respect to a Riesz basis $\left\{g_{i}\right\}_{i=1}^{\infty}$ if there exist a sequence of indexing sets $\left\{I_{i}\right\}_{i=1}^{\infty}$, positive constants $s>1$, and $C$ such that

$$
\left|\left\langle f_{k}, g_{j}\right\rangle\right| \leq \frac{C}{\sqrt{n}_{i}}(1+|i-j|)^{-s}
$$

and

$$
\left|\left\langle f_{k}, \tilde{g_{j}}\right\rangle\right| \leq \frac{C}{\sqrt{n}_{i}}(1+|i-j|)^{-s}
$$

for all $i, j \in \mathbb{N}$ and $k \in I_{i}$, where $\left\{\tilde{g}_{i}\right\}_{i=1}^{\infty}$ is the dual Riesz basis of $\left\{g_{i}\right\}_{i=1}^{\infty}$ and $n_{i}$ is the cardinality of $I_{i}$.

Note that if $\left\{g_{i}\right\}_{i=1}^{\infty}$ is an orthonomal basis for $H$ then the conditions (1) and (2) are identical and the conditions (3) and (4) are identical because $\left\{g_{i}\right\}_{i=1}^{\infty}=\left\{\tilde{g}_{i}\right\}_{i=1}^{\infty}$. Although we use the same terminology, our definitions are more general than Gröchenig's definition.

The following lemma is equivalent to the corresponding lemma in [7]. The only difference is the usage of exponential function. In [7], they use $e^{-\alpha t}, \alpha>0$, and in this paper we use $r^{t}, 0<r<1$, as exponential functions. 
Lemma 2.4. (1) For any positive constant $r<1$, there exists a constant $C>0$ such that

$$
\sum_{l=1}^{\infty} r^{|l-k|} r^{|l-j|} \leq C r^{\frac{1}{2}|k-j|}
$$

for all $k, j \in \mathbb{N}$.

(2) For any constant $s>1$, there exists a constant $C>0$ such that

$$
\sum_{l=1}^{\infty}(1+|l-k|)^{-s}(1+|l-j|)^{-s} \leq C(1+|\hat{k}-j|)^{-s}
$$

for all $k, j \in \mathbb{N}$.

Proof. (1) Fix $k, j \in \mathbb{N}$ and let $I_{1}=\left\{l \in \mathbb{N}:|l-j| \leq \frac{1}{2}|k-j|\right\}$ and $I_{2}=\mathbb{N}-I_{1}$. If $l \in I_{1}$, then $|l-k| \geq \frac{1}{2}|k-j|$. Hence, we have

$$
\begin{aligned}
\sum_{l=1}^{\infty} r^{|l-k|} r^{|l-j|} & =\sum_{l \in I_{1}} r^{|l-k|} r^{|l-j|}+\sum_{l \in I_{2}} r^{|l-k|} r^{|l-j|} \\
& \leq \sum_{l \in I_{1}} r^{\frac{1}{2}|k-j|} r^{|l-j|}+\sum_{l \in I_{2}} r^{|l-k|} r^{\frac{1}{2}|k-j|} \\
& =r^{\frac{1}{2}|k-j|}\left(\sum_{l \in I_{1}} r^{|l-j|}+\sum_{l \in I_{2}} r^{|l-k|}\right) \\
& \leq r^{\frac{1}{2}|k-j|}\left(\sum_{l=1}^{\infty} r^{|l-j|}+\sum_{l=1}^{\infty} r^{|l-k|}\right) \\
& =r^{\frac{1}{2}|k-j|}\left(\left(\sum_{l=1}^{j-1} r^{l}+\sum_{l=0}^{\infty} r^{l}\right)+\left(\sum_{l=1}^{k-1} r^{l}+\sum_{l=0}^{\infty} r^{l}\right)\right) \\
& \leq r^{\frac{1}{2}|k-j|} 2\left(\frac{r}{1-r}+\frac{1}{1-r}\right) \\
& =r^{\frac{1}{2}|k-j|} 2\left(\frac{1+r}{1-r}\right) \\
& =C r^{\frac{1}{2}|k-j|},
\end{aligned}
$$

where $C=2(1+r) /(1-r)$ and it is independent of $k$ and $j$. 
(2) Fix $k, j \in \mathbb{N}$ and let $I_{1}=\left\{l \in \mathbb{N}:|l-j| \leq \frac{1}{2}|k-j|\right\}$ and $I_{2}=\mathbb{N}-I_{1}$. If $l \in I_{1}$, then $|l-k| \geq \frac{1}{2}|k-j|$. Hence, we have

$$
\begin{aligned}
& \sum_{l=1}^{\infty}(1+|l-k|)^{-s}(1+|l-j|)^{-s} \\
= & \sum_{l \in I_{1}}(1+|l-k|)^{-s}(1+|l-j|)^{-s}+\sum_{l \in I_{2}}(1+|l-k|)^{-s}(1+|l-j|)^{-s} \\
\leq & \sum_{l \in I_{1}}\left(1+\frac{1}{2}|k-j|\right)^{-s}(1+|l-j|)^{-s} \\
& +\sum_{l \in I_{2}}(1+|l-k|)^{-s}\left(1+\frac{1}{2}|k-j|\right)^{-s} \\
= & \left(1+\frac{1}{2}|k-j|\right)^{-s}\left(\sum_{l \in I_{1}}(1+|l-j|)^{-s}+\sum_{l \in I_{2}}(1+|l-k|)^{-s}\right) \\
\leq & 2^{s}(1+|k-j|)^{-s}\left(\sum_{l \in I_{1}}(1+|l-j|)^{-s}+\sum_{l \in I_{2}}(1+|l-k|)^{-s}\right) \\
\leq & 2^{s}(1+|k-j|)^{-s}\left(\sum_{l=1}^{\infty}(1+|l-j|)^{-s}+\sum_{l=1}^{\infty}(1+|l-k|)^{-s}\right) \\
= & 2^{s}(1+|k-j|)^{-s}\left(\left(\sum_{l=1}^{j-1}(1+l)^{-s}+\sum_{l=0}^{\infty}(1+l)^{-s}\right)\right. \\
& \left.+\left(\sum_{l=1}^{k-1}(1+l)^{-s}+\sum_{l=0}^{\infty}(1+l)^{-s}\right)\right) \\
\leq & \left(2^{s}(1+|k-j|)^{-s}\right) 2\left(\int_{1}^{\infty} x^{-s} d x+\int_{1}^{\infty} x^{-s} d x+1\right) \\
= & \left(2^{s}(1+|k-j|)^{-s}\right)^{2}\left(\frac{s+1}{s-1}\right) \\
= & C(1+|k-j|)^{-s},
\end{aligned}
$$

where $C=2^{s+1}(s+1) /(s-1)$ and it is independent of $k$ and $j$.

\section{Main results}

Proposition 3.1. Let $\left\{f_{i}\right\}_{i=1}^{\infty}$ be a sequence in a Hilbert space $H$ and $\left\{g_{i}\right\}_{i=1}^{\infty}$ be a Riesz basis for $H$ with bounds $A, B$. If $\left\{f_{i}\right\}_{i=1}^{\infty}$ is 
exponentially or polynomially localized with respect to $\left\{g_{i}\right\}_{i=1}^{\infty}$, then $\left\{f_{i}\right\}_{i=1}^{\infty}$ is a Bessel sequence.

Proof. Since $\left\{g_{i}\right\}_{i=1}^{\infty}$ is a Riesz basis, we can write $f_{i}=\sum_{l=1}^{\infty}\left\langle f_{i}, \tilde{g}_{l}\right\rangle g_{l}$ for every $i \in \mathbb{N}$, where $\left\{\tilde{g}_{i}\right\}_{i=1}^{\infty}$ is the dual Riesz basis of $\left\{g_{i}\right\}_{i=1}^{\infty}$.

Let $f \in H$.

(1) Suppose that $\left\{f_{i}\right\}_{i=1}^{\infty}$ is exponentially localized. Then there exist a sequence of indexing sets $\left\{I_{k}\right\}_{k=1}^{\infty}$, positive constants $r<1$ and $C$ such that $\left|\left\langle f_{i}, g_{j}\right\rangle\right| \leq \frac{C}{\sqrt{n_{k}}} r^{|k-j|}$ and $\left|\left\langle f_{i}, \tilde{g}_{j}\right\rangle\right| \leq\left.\frac{C}{\sqrt{n_{k}}}\right|^{|k-j|}$ for all $k, j \in \mathbb{N}$ and $i \in I_{k}$, where $\left\{\tilde{g}_{i}\right\}_{i=1}^{\infty}$ is the dual Riesz basis of $\left\{g_{i}\right\}_{i=1}^{\infty}$ and $n_{k}$ is the cardinality of $I_{k}$. So, by Lemma 2.4 (1), we obtain

$$
\begin{aligned}
& \sum_{i=1}^{\infty}\left|\left\langle f, f_{i}\right\rangle\right|^{2}=\sum_{k=1}^{\infty} \sum_{i \in I_{k}}\left|\left\langle f, f_{i}\right\rangle\right|^{2} \\
& =\sum_{k=1}^{\infty} \sum_{i \in I_{k}}\left|\sum_{l=1}^{\infty}\left\langle f, g_{l}\right\rangle \overline{\left\langle f_{i}, \tilde{g}_{l}\right\rangle}\right|^{2} \\
& \leq \sum_{k=1}^{\infty} \sum_{i \in I_{k}}\left(\sum_{l=1}^{\infty}\left|\left\langle f, g_{l}\right\rangle\right|\left|\left\langle f_{i}, \tilde{g}_{l}\right\rangle\right|\right)^{2} \\
& \leq \sum_{k=1}^{\infty} \sum_{i \in I_{k}}\left(\sum_{l=1}^{\infty}\left|\left\langle f, g_{l}\right\rangle\right| \frac{C}{\sqrt{n_{k}}} r^{|k-l|}\right)^{2} \\
& =C^{2} \sum_{k=1}^{\infty}\left(\sum_{l=1}^{\infty}\left|\left\langle f, g_{l}\right\rangle\right| r^{|k-l|}\right)^{2} \\
& =C^{2} \sum_{k=1}^{\infty}\left(\sum_{l=1}^{\infty} \sum_{m=1}^{\infty}\left|\left\langle f, g_{l}\right\rangle\right|\left|\left\langle f, g_{m}\right\rangle\right| r^{|k-l|} r^{|k-m|}\right) \\
& =\left.C^{2} \sum_{l=1}^{\infty} \sum_{m=1}^{\infty}\left|\left\langle f, g_{l}\right\rangle\right|\left|\left\langle f, g_{m}\right\rangle\right| \sum_{k=1}^{\infty} r^{|k-l|}\right|^{|k-m|} \\
& \leq C^{2} C_{1} \sum_{l=1}^{\infty} \sum_{m=1}^{\infty}\left|\left\langle f, g_{l}\right\rangle\right|\left|\left\langle f, g_{m}\right\rangle\right| r^{\frac{|l-m|}{2}} \\
& \leq \frac{C^{2} C_{1}}{2} \sum_{l=1}^{\infty} \sum_{m=1}^{\infty}\left(\left|\left\langle f, g_{l}\right\rangle\right|^{2}+\left|\left\langle f, g_{m}\right\rangle\right|^{2}\right) r^{\frac{|l-m|}{2}} \\
& =\frac{C^{2} C_{1}}{2}\left(\sum_{l=1}^{\infty} \sum_{m=1}^{\infty} r^{\frac{|l-m|}{2}}\left|\left\langle f, g_{l}\right\rangle\right|^{2}+\sum_{m=1}^{\infty} \sum_{l=1}^{\infty} r^{\frac{|l-m|}{2}}\left|\left\langle f, g_{m}\right\rangle\right|^{2}\right)
\end{aligned}
$$




$$
\begin{aligned}
& \leq \frac{C^{2} C_{1}}{2}\left(\sum_{l=1}^{\infty}\left(\frac{1+\sqrt{r}}{1-\sqrt{r}}\right)\left|\left\langle f, g_{l}\right\rangle\right|^{2}+\sum_{m=1}^{\infty}\left(\frac{1+\sqrt{r}}{1-\sqrt{r}}\right)\left|\left\langle f, g_{m}\right\rangle\right|^{2}\right) \\
& =C^{2} C_{1} C_{2} \sum_{l=1}^{\infty}\left|\left\langle f, g_{l}\right\rangle\right|^{2} \\
& \leq C^{2} C_{1} C_{2} B\|f\|^{2},
\end{aligned}
$$

where $C_{1}=2(1+r) /(1-r)$ and $C_{2}=(1+\sqrt{r}) /(1-\sqrt{r})$. So,

$$
\sum_{i=1}^{\infty}\left|\left\langle f, f_{i}\right\rangle\right|^{2} \leq M\|f\|^{2}
$$

where $M=C^{2} C_{1} C_{2} B$. Therefore, $\left\{f_{i}\right\}_{i=1}^{\infty}$ is a Bessel sequence.

(2) Suppose that $\left\{f_{i}\right\}_{i=1}^{\infty}$ is polynomially localized. Then there exist a sequence of indexing sets $\left\{I_{k}\right\}_{k=1}^{\infty}$, positive constants $s>1$ and $C$ such that $\left|\left\langle f_{i}, g_{j}\right\rangle\right| \leq \frac{C}{\sqrt{n_{k}}}(1+|k-j|)^{-s}$ and $\left|\left\langle f_{i}, \tilde{g_{j}}\right\rangle\right| \leq \frac{C}{\sqrt{n_{k}}}(1+|k-j|)^{-s}$ for all $k, j \in \mathbb{N}$ and $i \in I_{k}$, where $\left\{\tilde{g}_{i}\right\}_{i=1}^{\infty}$ is the dual Riesz basis of $\left\{g_{i}\right\}_{i=1}^{\infty}$ and $n_{k}$ is the cardinality of $I_{k}$. So, by Lemma 2.4 (2), we obtain

$$
\begin{aligned}
& \sum_{i=1}^{\infty}\left|\left\langle f, f_{i}\right\rangle\right|^{2} \\
= & \sum_{k=1}^{\infty} \sum_{i \in I_{k}}\left|\left\langle f, f_{i}\right\rangle\right|^{2} \\
= & \sum_{k=1}^{\infty} \sum_{i \in I_{k}}\left|\sum_{l=1}^{\infty}\left\langle f, g_{l}\right\rangle \overline{\left\langle f_{i}, \tilde{g}_{l}\right\rangle}\right|^{2} \\
\leq & \sum_{k=1}^{\infty} \sum_{i \in I_{k}}\left(\sum_{l=1}^{\infty}\left|\left\langle f, g_{l}\right\rangle\right|\left|\left\langle f_{i}, \tilde{g}_{l}\right\rangle\right|\right)^{2} \\
\leq & \sum_{k=1}^{\infty} \sum_{i \in I_{k}}\left(\sum_{l=1}^{\infty}\left|\left\langle f, g_{l}\right\rangle\right| \frac{C}{\sqrt{n_{k}}}(1+|k-l|)^{-s}\right)^{2} \\
= & C^{2} \sum_{k=1}^{\infty}\left(\sum_{l=1}^{\infty}\left|\left\langle f, g_{l}\right\rangle\right|(1+|k-l|)^{-s}\right)^{2} \\
= & C^{2} \sum_{k=1}^{\infty}\left(\sum_{l=1}^{\infty} \sum_{m=1}^{\infty}\left|\left\langle f, g_{l}\right\rangle\right|\left|\left\langle f, g_{m}\right\rangle\right|(1+|k-l|)^{-s}(1+|k-m|)^{-s}\right)
\end{aligned}
$$




$$
\begin{aligned}
= & C^{2} \sum_{l=1}^{\infty} \sum_{m=1}^{\infty}\left|\left\langle f, g_{l}\right\rangle\right|\left|\left\langle f, g_{m}\right\rangle\right| \sum_{k=1}^{\infty}(1+|k-l|)^{-s}(1+|k-m|)^{-s} \\
\leq & C^{2} C_{1} \sum_{l=1}^{\infty} \sum_{m=1}^{\infty}\left|\left\langle f, g_{l}\right\rangle\right|\left|\left\langle f, g_{m}\right\rangle\right|(1+|l-m|)^{-s} \\
\leq & \frac{C^{2} C_{1}}{2} \sum_{l=1}^{\infty} \sum_{m=1}^{\infty}\left(\left|\left\langle f, g_{l}\right\rangle\right|^{2}+\left|\left\langle f, g_{m}\right\rangle\right|^{2}\right)(1+|l-m|)^{-s} \\
= & \frac{C^{2} C_{1}}{2}\left(\sum_{l=1}^{\infty} \sum_{m=1}^{\infty}(1+|l-m|)^{-s}\left|\left\langle f, g_{l}\right\rangle\right|^{2}\right. \\
& \left.+\sum_{m=1}^{\infty} \sum_{l=1}^{\infty}(1+|l-m|)^{-s}\left|\left\langle f, g_{m}\right\rangle\right|^{2}\right) \\
\leq & \frac{C^{2} C_{1}}{2}\left(\sum_{l=1}^{\infty}\left(\frac{s+1}{s-1}\right)\left|\left\langle f, g_{l}\right\rangle\right|^{2}+\sum_{m=1}^{\infty}\left(\frac{s+1}{s-1}\right)\left|\left\langle f, g_{l n}\right\rangle\right|^{2}\right) \\
= & C^{2} C_{1} C_{2} \sum_{l=1}^{\infty}\left|\left\langle f, g_{l}\right\rangle\right|^{2} \\
\leq & C^{2} C_{1} C_{2} B|| f \|^{2},
\end{aligned}
$$

where $C_{1}=2^{s+1}(s+1) /(s-1)$ and $C_{2}=(s+1) /(s-1)$. So,

$$
\sum_{i=1}^{\infty}\left|\left\langle f, f_{i}\right\rangle\right|^{2} \leq M\|f\|^{2}
$$

where $M=C^{2} C_{1} C_{2} B$. Therefore, $\left\{f_{i}\right\}_{i=1}^{\infty}$ is a Bessel sequence.

Corollary 3.2. Let $\left\{f_{i}\right\}_{i=1}^{\infty}$ be a sequence in a Hilbert space $H$ and $\left\{e_{i}\right\}_{i=1}^{\infty}$ be an orthonomal basis for $H$. If $\left\{f_{i}\right\}_{i=1}^{\infty}$ is exponentially or polynomially localized with respect to $\left\{e_{i}\right\}_{i=1}^{\infty}$, then $\left\{f_{i}\right\}_{i=1}^{\infty}$ is a Bessel sequence.

To prove Theorem 3.5 and Theorem 3.6, we need the well-known fact that a diagonally dominant matrix is invertible (see [9] for proof).

Lemma 3.3. Suppose that a matrix $A=\left(a_{i j}\right)_{i, j=1}^{\infty}$ defines a bounded self-adjoint operator on $l^{2}(\mathbb{N})$ and that $A$ satisfies the condition of diagonal dominance for every $i \in \mathbb{N}$; that is, there exists a positive constant $\delta$ such that

$$
\left|a_{i i}\right|-\sum_{j: j \neq i}\left|a_{i j}\right| \geq \delta
$$


for every $i \in \mathbb{N}$. Then $A$ is invertible on $l^{2}(\mathbb{N})$.

If $\left\{f_{i}\right\}_{i=1}^{\infty}$ is a Bessel sequence, we can define a bounded operator $T$, usually called the pre-frame operator associated to $\left\{f_{i}\right\}_{i=1}^{\infty}$ :

$$
T: l^{2}(\mathbb{N}) \rightarrow H, \quad T\left\{c_{i}\right\}_{i=1}^{\infty}=\sum_{i=1}^{\infty} c_{i} f_{i} .
$$

Then its adjoint $T^{*}: H \rightarrow l^{2}(\mathbb{N})$ is defined by $T^{*} f=\left\{\left\langle f, f_{i}\right\rangle\right\}_{i=1}^{\infty}$ for every $f \in H$. By composing $T$ and $T^{*}$, we obtain the frame operator $S$ :

$$
S: H \rightarrow H, \quad S f=T T^{*} f=\sum_{i=1}^{\infty}\left\langle f, f_{i}\right\rangle f_{i} .
$$

If $\left\{f_{i}\right\}_{i=1}^{\infty}$ is a Bessel sequence, the series defining $S$ converges unconditionally for all $f \in H$, and $S$ is a bounded self-adjoint operator on $H$.

THEOREM 3.4. Let $\left\{f_{i}\right\}_{i=1}^{\infty}$ be a Bessel sequence in a Hilbert space $H$ and $\left\{g_{i}\right\}_{i=1}^{\infty}$ be a Riesz basis for $H$. If the matrix $\left\{\left\langle S g_{i}, g_{j}\right\rangle\right\}_{i, j=1}^{\infty}$ is invertible on $l^{2}(\mathbb{N})$, then $\left\{f_{i}\right\}_{i=1}^{\infty}$ is a frame for $H$.

The proof of Theorem 3.4 can be found in [10].

THEOREM 3.5. Let $\left\{f_{i}\right\}_{i=1}^{\infty}$ be a sequence in a Hilbert space $H$ and $\left\{g_{i}\right\}_{i=1}^{\infty}$ be a Riesz basis for $H$, and suppose that $\left\{f_{i}\right\}_{i=1}^{\infty}$ is exponentially localized with respect to $\left\{g_{i}\right\}_{i=1}^{\infty}$; that is, there exist a sequence of indexing sets $\left\{I_{i}\right\}_{i=1}^{\infty}$, positive constants $r<1$, and $C_{1}$ such that

$$
\left|\left\langle f_{k}, g_{j}\right\rangle\right| \leq\left.\frac{C_{1}}{\sqrt{n_{i}}}\right|^{|i-j|}
$$

and

$$
\left|\left\langle f_{k}, \tilde{g}_{j}\right\rangle\right| \leq\left.\frac{C_{1}}{\sqrt{n_{i}}}\right|^{|i-j|}
$$

for all $i, j \in \mathbb{N}$ and $k \in I_{i}$, where $\left\{\tilde{g}_{i}\right\}_{i=1}^{\infty}$ is the dual Riesz basis of $\left\{g_{i}\right\}_{i=1}^{\infty}$ and $n_{i}$ is the cardinality of $I_{i}$.

If there exists a positive constant $C_{2}$ such that

$$
\sum_{i=1}^{\infty}\left|\left\langle f_{i}, g_{j}\right\rangle\right|^{2} \geq C_{2}^{2}
$$

and

then $\left\{f_{i}\right\}_{i=1}^{\infty}$ is a frame for $H$.

$$
\sqrt{2} C_{2}>\left(\frac{1+r}{1-r}\right) C_{1}
$$


Proof. By Proposition 3.1, $\left\{f_{i}\right\}_{i=1}^{\infty}$ is a Bessel sequence. So it suffices to show that $\left\{f_{i}\right\}_{i=1}^{\infty}$ has a lower frame bound. Fix $i \in \mathbb{N}$ and consider

$$
\left|\left\langle S g_{i}, g_{i}\right\rangle\right|-\sum_{j: j \neq i}\left|\left\langle S g_{i}, g_{j}\right\rangle\right| .
$$

Since $\left\langle S g_{i}, g_{j}\right\rangle=\sum_{k=1}^{\infty}\left\langle g_{i}, f_{k}\right\rangle\left\langle f_{k}, g_{j}\right\rangle$ and $\left\langle S g_{i}, g_{i}\right\rangle==\sum_{k=1}^{\infty}\left|\left\langle g_{i}, f_{k}\right\rangle\right|^{2}$, we have

$$
\begin{aligned}
\sum_{j: j \neq i}\left|\left\langle S g_{i}, g_{j}\right\rangle\right| & =\sum_{j=1}^{\infty}\left|\left\langle S g_{i}, g_{j}\right\rangle\right|-\left|\left\langle S g_{i}, g_{i}\right\rangle\right| \\
& \leq \sum_{j=1}^{\infty} \sum_{k=1}^{\infty}\left|\left\langle g_{i}, f_{k}\right\rangle\right|\left|\left\langle f_{k}, g_{j}\right\rangle\right|-\sum_{k=1}^{\infty}\left|\left\langle g_{i}, f_{k}\right\rangle\right|^{2} \\
& \leq \sum_{j=1}^{\infty} \sum_{k=1}^{\infty}\left|\left\langle g_{i}, f_{k}\right\rangle\right|\left|\left\langle f_{k}, g_{j}\right\rangle\right|-C_{2}^{2} .
\end{aligned}
$$

Since $\left\{f_{i}\right\}_{i=1}^{\infty}$ is exponentially localized with respect to $\left\{g_{i}\right\}_{i=1}^{\infty}$,

$$
\begin{aligned}
\sum_{k=1}^{\infty}\left|\left\langle g_{i}, f_{k}\right\rangle\right|\left|\left\langle f_{k}, g_{j}\right\rangle\right| & =\sum_{m=1}^{\infty} \sum_{k \in I_{m}}\left|\left\langle g_{i}, f_{k}\right\rangle\right|\left|\left\langle f_{k}, g_{j}\right\rangle\right| \\
& \leq C_{1}^{2} \sum_{m=1}^{\infty} \sum_{k \in I_{m}}\left(\frac{r^{|i-m|}}{\sqrt{n_{m}}}\right)\left(\frac{r^{|j-m|}}{\sqrt{n_{m}}}\right) \\
& =\left.C_{1}^{2} \sum_{m=1}^{\infty} r^{|i-m|}\right|^{|j-m|} .
\end{aligned}
$$

From this inequality, we obtain

$$
\begin{aligned}
\sum_{j=1}^{\infty} \sum_{k=1}^{\infty}\left|\left\langle g_{i}, f_{k}\right\rangle\right|\left|\left\langle f_{k}, g_{j}\right\rangle\right| & \leq C_{1}^{2} \sum_{m=1}^{\infty} \sum_{j=1}^{\infty} r^{|i-m|} r^{|j-m|} \\
& =C_{1}^{2} \sum_{m=1}^{\infty} r^{|i-m|} \sum_{j=1}^{\infty} r^{|j-m|} \\
& \leq C_{1}^{2}\left(\frac{r}{1-r}+\frac{1}{1-r}\right)^{2} \\
& =C_{1}^{2}\left(\frac{1+r}{1-r}\right)^{2} .
\end{aligned}
$$


Hence,

$$
\sum_{j: j \neq i}\left|\left\langle S g_{i}, g_{j}\right\rangle\right| \leq C_{1}^{2}\left(\frac{1+r}{1-r}\right)^{2}-C_{2}^{2}
$$

Finally,

$$
\left|\left\langle S g_{i}, g_{i}\right\rangle\right|-\sum_{j: j \neq i}\left|\left\langle S g_{i}, g_{j}\right\rangle\right| \geq 2 C_{2}^{2}-C_{1}^{2}\left(\frac{1+r}{1-r}\right)^{2}>0,
$$

where the constants $r, C_{1}, C_{2}$ are independent of $i$. Therefore, $\left\{f_{i}\right\}_{i=1}^{\infty}$ is a frame for $H$.

THEOREM 3.6. Let $\left\{f_{i}\right\}_{i=1}^{\infty}$ be a sequence in a Hilbert space $H$ and $\left\{g_{i}\right\}_{i=1}^{\infty}$ be a Riesz basis for $H$, and suppose that $\left\{f_{i}\right\}_{i=1}^{\infty}$ is polynomially localized with respect to $\left\{g_{i}\right\}_{i=1}^{\infty}$; that is, there exists a sequence of indexing sets $\left\{I_{i}\right\}_{i=1}^{\infty}$, positive constants $s>1$, and $C_{1}$ such that

$$
\left|\left\langle f_{k}, g_{j}\right\rangle\right| \leq \frac{C_{1}}{\sqrt{n_{i}}}(1+|i-j|)^{-s}
$$

and

$$
\left|\left\langle f_{k}, \tilde{g}_{j}\right\rangle\right| \leq \frac{C_{1}}{\sqrt{n_{i}}}(1+|i-j|)^{-s}
$$

for all $i, j \in \mathbb{N}$ and $k \in I_{i}$ where $\left\{\tilde{g}_{i}\right\}_{i=1}^{\infty}$ is the dual Riesz basis of $\left\{g_{i}\right\}_{i=1}^{\infty}$ and $n_{i}$ is the cardinality of $I_{i}$.

If there exists a positive constant $C_{2}$ such that

$$
\sum_{i=1}^{\infty}\left|\left\langle f_{i}, g_{j}\right\rangle\right|^{2} \geq C_{2}^{2}
$$

and

$$
\sqrt{2} C_{2}>\left(\frac{s+1}{s-1}\right) C_{1}
$$

then $\left\{f_{i}\right\}_{i=1}^{\infty}$ is a frame for $H$.

Proof. By Proposition 3.1, $\left\{f_{i}\right\}_{i=1}^{\infty}$ is a Bessel sequence. So it suffices to show that $\left\{f_{i}\right\}_{i=1}^{\infty}$ has a lower frame bound. Fix $i \in \mathbb{N}$ and consider

$$
\left|\left\langle S g_{i}, g_{i}\right\rangle\right|-\sum_{j: j \neq i}\left|\left\langle S g_{i}, g_{j}\right\rangle\right|
$$


Since $\left\langle S g_{i}, g_{j}\right\rangle=\sum_{k=1}^{\infty}\left\langle g_{i}, f_{k}\right\rangle\left\langle f_{k}, g_{j}\right\rangle$ and $\left\langle S g_{i}, g_{i}\right\rangle=\sum_{k=1}^{\infty}\left|\left\langle g_{i}, f_{k}\right\rangle\right|^{2}$, we have

$$
\begin{aligned}
\sum_{j: j \neq i}\left|\left\langle S g_{i}, g_{j}\right\rangle\right| & =\sum_{j=1}^{\infty}\left|\left\langle S g_{i}, g_{j}\right\rangle\right|-\left|\left\langle S g_{i}, g_{i}\right\rangle\right| \\
& \leq \sum_{j=1}^{\infty} \sum_{k=1}^{\infty}\left|\left\langle g_{i}, f_{k}\right\rangle\right|\left|\left\langle f_{k}, g_{j}\right\rangle\right|-\sum_{k=1}^{\infty}\left|\left\langle g_{i}, f_{k}\right\rangle\right|^{2} \\
& \leq \sum_{j=1}^{\infty} \sum_{k=1}^{\infty}\left|\left\langle g_{i}, f_{k}\right\rangle\right|\left|\left\langle f_{k}, g_{j}\right\rangle\right|-C_{2}^{2}
\end{aligned}
$$

Since $\left\{f_{i}\right\}_{i=1}^{\infty}$ is polynomially localized with respect to $\left\{g_{i}\right\}_{i=1}^{\infty}$,

$$
\begin{aligned}
& \sum_{k=1}^{\infty}\left|\left\langle g_{i}, f_{k}\right\rangle\right|\left|\left\langle f_{k}, g_{j}\right\rangle\right| \\
= & \sum_{m=1}^{\infty} \sum_{k \in I_{m}}\left|\left\langle g_{i}, f_{k}\right\rangle\right|\left|\left\langle f_{k}, g_{j}\right\rangle\right| \\
\leq & C_{1}^{2} \sum_{m=1}^{\infty} \sum_{k \in I_{m}}\left(\frac{(1+|i-m|)^{-s}}{\sqrt{n_{m}}}\right)\left(\frac{(1+|j-m|)^{-s}}{\sqrt{n_{m}}}\right) \\
= & C_{1}^{2} \sum_{m=1}^{\infty}(1+|i-m|)^{-s}(1+|j-m|)^{-s} .
\end{aligned}
$$

From these inequalities, we obtain

$$
\begin{aligned}
\sum_{j=1}^{\infty} \sum_{k=1}^{\infty}\left|\left\langle g_{i}, f_{k}\right\rangle\right|\left|\left\langle f_{k}, g_{j}\right\rangle\right| & \leq \sum_{m=1}^{\infty}(1+|i-m|)^{-s} \sum_{j=1}^{\infty}(1+|j-m|)^{-s} \\
& \leq C_{1}^{2}\left(\frac{s+1}{s-1}\right)^{2} .
\end{aligned}
$$

Hence,

Finally,

$$
\sum_{j: j \neq i}\left|\left\langle S g_{i}, g_{j}\right\rangle\right| \leq C_{1}^{2}\left(\frac{s+1}{s-1}\right)^{2}-C_{2}^{2}
$$

$$
\left|\left\langle S g_{i}, g_{i}\right\rangle\right|-\sum_{j: j \neq i}\left|\left\langle S g_{i}, g_{j}\right\rangle\right| \geq 2 C_{2}^{2}-C_{1}^{2}\left(\frac{s+1}{s-1}\right)^{2}>0
$$

where the constants $r, C_{1}, C_{2}$ are independent of $i$. Therefore, $\left\{f_{i}\right\}_{i=1}^{\infty}$ is a frame for $H$. 


\section{Examples}

As an application of our main theorem, we show examples. The first example is a sequence which is exponentially localized with respect to an orthonormal basis $\left\{e_{i}\right\}_{i=1}^{\infty}$ for $H$.

EXAMPLE 4.1. Let $0<r \leq \frac{1}{6}$,

$$
\begin{aligned}
& f_{1}=e_{1}+r e_{2}+r^{2} e_{3}+\cdots, \\
& f_{2}=\frac{1}{\sqrt{2}}\left(r e_{1}+e_{2}+r e_{3}+r^{2} e_{4}+\cdots\right), \\
& f_{3}=\frac{1}{\sqrt{2}}\left(r e_{1}+e_{2}+r e_{3}+r^{2} e_{4}+\cdots\right), \\
& f_{4}=\frac{1}{\sqrt{3}}\left(r^{2} e_{1}+r e_{2}+e_{3}+r e_{4}+r^{2} e_{5}+\cdots\right), \\
& f_{5}=\frac{1}{\sqrt{3}}\left(r^{2} e_{1}+r e_{2}+e_{3}+r e_{4}+r^{2} e_{5}+\cdots\right), \\
& f_{6}=\frac{1}{\sqrt{3}}\left(r^{2} e_{1}+r e_{2}+e_{3}+r e_{4}+r^{2} e_{5}+\cdots\right),
\end{aligned}
$$

and $I_{i}=\left\{\frac{i(i-1)}{2}+1, \frac{i(i-1)}{2}+2, \ldots, \frac{i(i-1)}{2}+i\right\}$ for each $i \in \mathbb{N}$. Then $\left\{I_{i}\right\}_{i=1}^{\infty}$ is a sequence of indexing sets and $n_{i}=\left|I_{i}\right|=i$.

For every $i, j \in \mathbb{N}$ and $k \in I_{i}$,

$$
\left|\left\langle f_{k}, e_{j}\right\rangle\right|=\frac{1}{\sqrt{i}} r^{|i-j|}
$$

and

$$
\sum_{i=1}^{\infty}\left|\left\langle f_{i}, e_{j}\right\rangle\right|^{2}=\left(r^{j-1}\right)^{2}+\left(r^{j-2}\right)^{2}+\cdots+r^{2}+1+r^{2}+r^{4}+\cdots \geq 1 .
$$

Let $C_{1}=1$ and $C_{2}=1$. Then,

$$
\sum_{i=1}^{\infty}\left|\left\langle f_{i}, e_{j}\right\rangle\right|^{2} \geq C_{2}^{2}
$$

and

$$
\sqrt{2} C_{2}=\sqrt{2}>\frac{7}{5}=\frac{1+\frac{1}{6}}{1-\frac{1}{6}} \geq\left(\frac{1+r}{1-r}\right) C_{1} .
$$

Hence, $\left\{f_{i}\right\}_{i=1}^{\infty}$ is a frame for $H$. 
The second example is a sequence which is polynomially localized with respect to an orthonormal basis $\left\{e_{i}\right\}_{i=1}^{\infty}$ for $H$.

EXAMPLE 4.2. Let $s \geq 6$,

$$
\begin{aligned}
f_{1} & =e_{1}+2^{-s} e_{2}+3^{-s} e_{3}+\cdots \\
f_{2} & =\frac{1}{\sqrt{2}}\left(2^{-s} e_{1}+e_{2}+2^{-s} e_{3}+3^{-s} e_{4}+\cdots\right), \\
f_{3} & =\frac{1}{\sqrt{2}}\left(2^{-s} e_{1}+e_{2}+2^{-s} e_{3}+3^{-s} e_{4}+\cdots\right), \\
f_{4} & =\frac{1}{\sqrt{3}}\left(3^{-s} e_{1}+2^{-s} e_{2}+e_{3}+2^{-s} e_{4}+3^{-s} e_{5}+\cdots\right), \\
f_{5} & =\frac{1}{\sqrt{3}}\left(3^{-s} e_{1}+2^{-s} e_{2}+e_{3}+2^{-s} e_{4}+3^{-s} e_{5}+\cdots\right), \\
f_{6} & =\frac{1}{\sqrt{3}}\left(3^{-s} e_{1}+2^{-s} e_{2}+e_{3}+2^{-s} e_{4}+3^{-s} e_{5}+\cdots\right) \\
\ldots &
\end{aligned}
$$

and $I_{i}=\left\{\frac{i(i-1)}{2}+1, \frac{i(i-1)}{2}+2, \ldots, \frac{i(i-1)}{2}+i\right\}$ for each $i \in \mathbb{N}$. Then for every $i, j \in \mathbb{N}$ and $k \in I_{i}$,

$$
\left|\left\langle f_{k}, e_{j}\right\rangle\right|=\frac{1}{\sqrt{i}}(1+|i-j|)^{-s},
$$

and

$$
\begin{aligned}
\sum_{i=1}^{\infty}\left|\left\langle f_{i}, e_{j}\right\rangle\right|^{2}= & \left(j^{-s}\right)^{2}+\left((j-1)^{-s}\right)^{2}+\cdots \\
& +\left(2^{-s}\right)^{2}+1+\left(2^{-s}\right)^{2}+\left(3^{-s}\right)^{2}+\cdots \geq 1 .
\end{aligned}
$$

Let $C_{1}=1$ and $C_{2}=1$. Then,

$$
\sum_{i=1}^{\infty}\left|\left\langle f_{i}, e_{j}\right\rangle\right|^{2} \geq C_{2}^{2}
$$

and

Hence, $\left\{f_{i}\right\}_{i=1}^{\infty}$ is a frame for $H$.

$$
\sqrt{2} C_{2}=\sqrt{2}>\frac{7}{5}=\frac{6+1}{6-1} \geq\left(\frac{s+1}{s-1}\right) C_{1}
$$

REMARK. In [5], Gröchenig gave more general definition of localization than in [7]. Essentially, his definition allows only bounded number of elements to be localized at each vector in the given Riesz basis. But, our definition sets no bound for the number of localized elements. In 
Example 4.1 and Example 4.2, the number of localized elements $f_{i}$ for each $g_{j}$ is unbounded. So Gröchenig's definition is not applicable to these examples.

\section{References}

[1] P. G. Casazza and O. Christensen, Approximation of the inverse frame opertor and applications to Gabor frames, J. Approx. Theory 103 (2000), 338-356.

[2] O. Christensen, An introduction to frames and Riesz basis, Birkhäuser, Boston, 2003.

[3] _ A Paley-Wiener theorem for frames, Proc. Amer. Math. Soc. 123 (1995), 2199-2201.

[4] _ Frame perturbations, Proc. Amer. Math. Soc. 123 (1995), 1217-1220.

[5] E. Cordero and K. Gröchenig, Localization of frames II, Appl. Comput. Harmon. Anal. 17 (2004), 29-47.

[6] I. Daubechies, Ten Lectures on Wavelets, SIAM Conference Series in Applied Mathematiccs, SIAM, Boston, 1992.

[7] K. Gröchenig, Localization of frames, Banach frames, and the invertiblility of the frame operator, J. Fourier Anal. Appl. 10 (2004), 105-132.

[8] _ Localized frames are finite unions of Riesz sequences, Adv. Comput. Math. 18 (2003), 149-157.

[9] _ Foundations of time-frequency analysis, Birkhäuser, Boston, 2001.

[10] Y. H. Ha and J. Y. Ryu, Localization property and frames, Honam Math. J. 27 (2005), 233-241.

[11] R. Young, An Introduction to Nonharmonic Fourier Series, Academic Press, NewYork, 1980.

Department of Mathematics

Ajou University

Suwon 442-749, Korea

E-mail: yhha@ajou.ac.kr

juyeonryu@ajou.ac.kr 